\title{
Selective hydroxylation of carbon surfaces for long- lasting hydrophilicity by a green chemistry process
}

\author{
Madeleine Wilsey \\ University of Rochester \\ Kendra Watson \\ University of Rochester \\ Omolade Fasusi \\ University of Rochester \\ Brian Yegela \\ University of Rochester \\ Connor Cox \\ University of Rochester \\ Patrick Raffaele \\ University of Rochester \\ Astrid Mueller ( $\nabla$ astrid.mueller@rochester.edu ) \\ University of Rochester https://orcid.org/0000-0002-2785-6808
}

\section{Article}

Keywords:

Posted Date: February 2nd, 2022

DOI: https://doi.org/10.21203/rs.3.rs-1284564/v1

License: (c) (i) This work is licensed under a Creative Commons Attribution 4.0 International License.

Read Full License 


\section{Selective hydroxylation of carbon surfaces for long-}

\section{lasting hydrophilicity by a green chemistry process}

Madeleine K. Wilsey, ${ }^{1}$ Kendra R. Watson, ${ }^{2,4}$ Omolade C. Fasusi, ${ }^{2,4}$ Brian P. Yegela, ${ }^{2,4}$ Connor P. Cox, ${ }^{1}$ Patrick R. Raffaele, ${ }^{2}$ Astrid M. Müller ${ }^{* 1,2,3}$

${ }^{1}$ Materials Science Program, University of Rochester, Rochester, New York 14627, United States. ${ }^{2}$ Department of Chemical Engineering, University of Rochester, Rochester, New York 14627, United States. ${ }^{3}$ Department of Chemistry, University of Rochester, Rochester, New York 14627, United States.

${ }^{4}$ These authors contributed equally: Kendra R. Watson, Omolade C. Fasusi, and Brian P. Yegela

*Corresponding author email: astrid.mueller@rochester.edu

\section{Abstract}

We report the selective hydroxylation of carbon surfaces that rendered initially hydrophobic carbon fiber paper hydrophilic for more than five months. This long time of sustained hydrophilicity is unprecedented. Carbon fiber paper is an inexpensive, electrically conductive, high surface area material that is additionally nontoxic, biocompatible, robust, and scalable. But its hydrophobicity prevents widespread use in aqueous applications. Inhibition of overoxidation of carbon beyond hydroxylation is especially challenging because the first oxidation step is thermodynamically most difficult and subsequent oxidations are much easier. We achieved 
selectivity for less oxidized hydroxyls over carboxyls by an environmentally friendly, solutionprocessable, acid-free carbon surface functionalization treatment that is rapid, amenable to large scale applications, and did not damage carbon fibers and their network architectures. The development of this mild, green chemistry carbon surface treatment that provides selectivity for surface hydroxyls transforms the utility of carbon materials.

\section{Introduction}

Carbon materials are electrically conductive, inexpensive, nontoxic, robust, scalable, and chemically inert. They can also possess high surface area, such as carbon fiber paper (CFP), making them desirable in many electrochemical, fuel cell, ${ }^{1,2}$ electrolyzer, ${ }^{3,4}$ supercapacitor, ${ }^{5-10}$ sensing, ${ }^{11,12}$ water desalination ${ }^{13}$ and wastewater treatment ${ }^{14,15}$ applications. Another advantage is the excellent biocompatibility of CFP for in-vitro and in-vivo studies, ${ }^{16}$ for biosensing applications, ${ }^{17,} 18$ tissue engineering, ${ }^{19}$ and in regenerative medicine and cancer treatment. ${ }^{20}$ Globally scalable clean energy ${ }^{21-23}$ and water purification ${ }^{24,} 25$ technologies as well as biocompatible systems must operate in aqueous media and, therefore, require carbon support materials that are hydrophilic for long periods of time.

One obstacle that inhibits widespread use of carbon materials in aqueous systems is their high hydrophobicity. Hydrophilic carbon surfaces are needed to take advantage of the high internal surface area of CFP in aqueous media. Any hydrophilicity-imparting treatment must leave the carbon fiber network structurally intact, so that specifically designed carbon network architectures and pore sizes, which critically affect mass transport characteristics, are not altered during the process. 
Here, we report selective carbon surface functionalization with hydroxyl groups that imparted long-lasting hydrophilicity to CFP without damaging the carbon fibers and their network architectures. Treatment of CFP in $1 \mathrm{M}$ aqueous sodium dodecyl sulfate (SDS) solution followed by electrooxidation in $0.1 \mathrm{M}$ aqueous potassium bicarbonate electrolyte took less than 30 minutes of preparation time and imparted excellent wettability by water (Fig. 1). CFP remained hydrophilic for 31 days when stored in ambient air and more than 5 months upon storage under water. Systematic iterations of thirteen different chemical treatments together with X-ray photoelectron spectra showed that surface hydroxyls, not carboxyls, were predictive of sustained hydrophilicity. Our finding explains why previously reported, harsher treatments with strong acids or high temperatures did not generate CFP with long-lived hydrophilicity. More potent oxidants are capable of oxidizing carbon all the way to carboxyls, whereas our acid-free, more benign SDSelectrooxidation treatment provided selectivity for less oxidized surface hydroxyl groups without detrimental overoxidation.

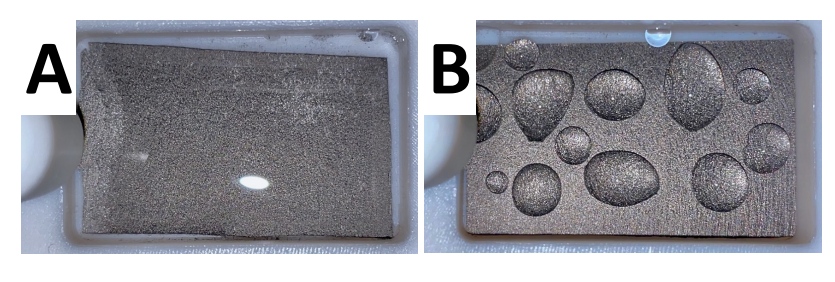

Fig. 1. Wettability of treated and untreated CFP by water. A Photograph of hydrophilic, SDS-electrooxidation treated CFP, which was wetted well by water. The white spot is a reflection from the room light. B Photograph of hydrophobic, untreated CFP, which remained unwetted by water. A custom-made Teflon tub served as barrier against water spillover.

Previously reported methods to increase hydrophilicity of CFP are plasma or chemical etching techniques, sometimes in combination with heat treatments. Ammonia, ${ }^{14}$ nitrogen, ${ }^{26}$ oxygen ${ }^{13,27-}$ ${ }^{29}$ or air ${ }^{30}$ plasma etching protocols have been reported. Such plasma etches work reasonably well 
on a laboratory scale, where electrode sizes are on the order of square centimeters or less; yet, plasma methods lead to significant embrittlement of CFP, precluding the production of structurally stable, large area CFP sheets that are needed for industrial scale electrolyzers and other applications. Ozone treatment also oxidizes carbon surfaces for improved hydrophilicity. ${ }^{31,32} \mathrm{An}$ additional disadvantage is that plasma and ozone generators require large capital expense, especially for large area substrates. Thermal oxidation by a treatment that took more than 16 hours also increased hydrophilicity, ${ }^{33}$ but that lasted only for 2 days when we followed the described procedure; significant damage to the carbon fibers was also observed (Fig. S1). The long preparation time hampers most applications and is not conducive for commercial viability.

We attempted a few more thermal oxidations in ambient air by annealing CFP at $500{ }^{\circ} \mathrm{C}$ for 5 or 10 minutes or at $800{ }^{\circ} \mathrm{C}$ for $1.5,3$ or 5 minutes. All CFP samples that were annealed at $500{ }^{\circ} \mathrm{C}$ were hydrophobic, whereas pretreated CFP that was annealed at $800{ }^{\circ} \mathrm{C}$ for 5 minutes remained hydrophilic for four days when stored in ambient air. All heat treatments led to extremely brittle CFP; in fact, some CFP pieces disintegrated altogether upon heat treatment. Scanning electron microscopy images revealed significant damage to the carbon fibers (Fig. S1).

Solution-processable modifications are a more cost-effective alternative. Reported chemical treatments required concentrated mineral acids and were tedious. $6,7,10,12,34-38$ They raised hydrophilicity, but materials were further functionalized immediately, indicating that hydrophilicity of carbon surfaces did not last. A hydrothermal process taking 12 hours was reported for CFP activation for further impregnation by salicylic acid. ${ }^{11}$ Hydrothermal methods require expensive autoclaves, whose scaleup for large area CFP sheets is inherently difficult and limited by the safety and design of reactors. The long preparation time of this hydrothermal process is another obstacle for many applications. 
Another approach to enhance CFP hydrophilicity is electro-etching. We found two reports on electro-etched CFP in the literature; both procedures required strong mineral acids and resulted in significant damage to the carbon fibers. ${ }^{39,} 40$

Our new green chemistry CFP treatment functionalized carbon surfaces by selective hydroxylation, resulting in long-lasting hydrophilicity, without embrittlement and structural damage to carbon fibers and their network architectures. The method solely uses solutionprocessable operations, takes less than 30 minutes of preparation time, and is suitable for largescale manufacturing.

\section{Results and discussion}

Green chemistry process for carbon surface functionalization. We selectively modified carbon surfaces with hydroxyl groups, which imparted long-lasting hydrophilicity to carbon fiber paper (CFP) without damaging the carbon fiber network. Our acid-free aqueous process consisted of sonication of CFP in $1 \mathrm{M}$ sodium dodecyl sulfate (SDS) solution, followed by electrooxidation in $0.1 \mathrm{M} \mathrm{pH} 8.7$ aqueous $\mathrm{KHCO}_{3}$ electrolyte at $+1.63 \mathrm{~V}$ vs. $\mathrm{Ag} / \mathrm{AgCl}$ for 20 minutes. This environmentally benign treatment prevented overoxidation of surface carbon and resulted in sustained hydrophilicity for 31 days in ambient air and more than 5 months upon storage under water.

All previously described chemical procedures to make CFP hydrophilic include the use of strong mineral acids. Our acid-free approach paves the way for creating large areas of hydrophilic CFP via a benign, green treatment, without the need for harsh acids and concomitant environmental and safety issues. Importantly, carbon fibers remained intact and smooth upon the acid-free treatment; no fraying of or damage to carbon fibers was observed in scanning electron microscopy (SEM) 
images (Fig. 2A-B). This finding is supported by visible inspection; photographs of CFP before and after SDS-electrooxidation treatment looked virtually identical, with no darkening upon treatment (Fig. 2C). In general, fraying and breaking of carbon fibers lead to a darkened appearance of CFP because of increased internal scattering of ambient light at the rough carbon surfaces within the fiber network. For comparison, we repeated the only reported literature procedures that used electrooxidation to impart hydrophilicity, as published by Wang et al. ${ }^{39}$ and Kazemi et al. ${ }^{40}$ We took SEM images and photographs that revealed that both procedures, which employed mineral acid electrolytes, resulted in significantly damaged carbon fibers (Fig. 2F-G). Such damage to the structural integrity of carbon fibers detrimentally affects mass transport characteristics and creates breaking points within CFP sheets, which lower their mechanical stability. Clearly, our acid-free, more benign treatment resulted in long-lasting hydrophilicity without damaging the carbon fibers and their network, which led to excellent bendability, compared to untreated CFP (Fig. 2D-E). We were unable to bend CFP that was treated such that SEM images showed significant damage to carbon fibers; those CFP pieces broke immediately upon application of a bending moment. 

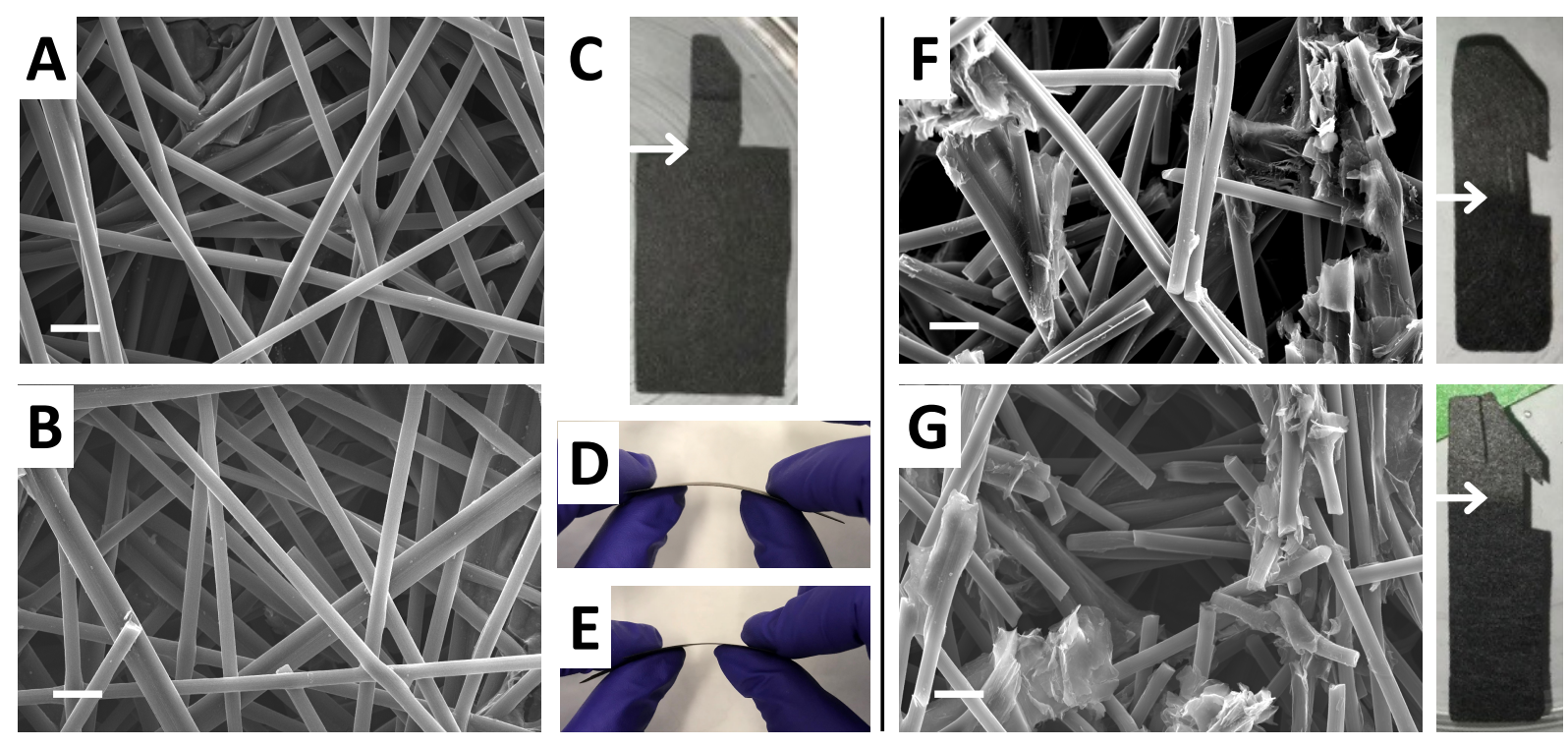

Fig. 2. Comparison of the acid-free, aqueous, green chemistry process with reported electrooxidation procedures to impart hydrophilicity to CFP. A SEM image of untreated CFP. B SEM image of SDS-electrooxidation treated CFP. C Photograph of untreated CFP (above white arrow) and SDS-electrooxidation treated CFP (below white arrow); the crease in the untreated CFP part stems from clamping the CFP in the electrooxidation cell. D, E Photographs of bending of untreated CFP (D) and SDS-electrooxidation treated CFP (E). F, G SEM images (left) and photographs (right) of CFP treated by the only reported electrooxidation procedures, which required mineral acids; F CFP treated according to Wang et al. ${ }^{39} \mathbf{G}$ CFP treated according to Kazemi et al. ${ }^{40}$

Systematic iterations of carbon surface modifications. In the following, we show that our acid-free method selectively hydroxylated carbon surfaces without overoxidation. To this end, and in keeping with many literature reports that make use of strong acids to install hydrophilic surface functional groups on CFP, we additionally assessed twelve acid-containing treatments to provide systematic iterations of carbon surface modifications. Acid-containing treatments started with a 
chemical etch in strongly oxidizing piranha solution, a 4:1 (v/v) mixture of concentrated sulfuric acid and 30\% aqueous hydrogen peroxide solution, possibly followed by a second chemical etch (1 $\mathrm{M}$ aqueous SDS or $6 \mathrm{M}$ aqueous $\mathrm{KOH}$ solution, or concentrated formic acid, or none) and electrooxidation in $0.1 \mathrm{M}$ aqueous $\mathrm{KHCO}_{3}$ electrolyte at $+1.63 \mathrm{~V}$ vs. $\mathrm{Ag} / \mathrm{AgCl}$ for 20 minutes (Fig. 3A). For comparison, the simpler, more rapid, acid-free procedure is outlined in Fig. 3B.

A

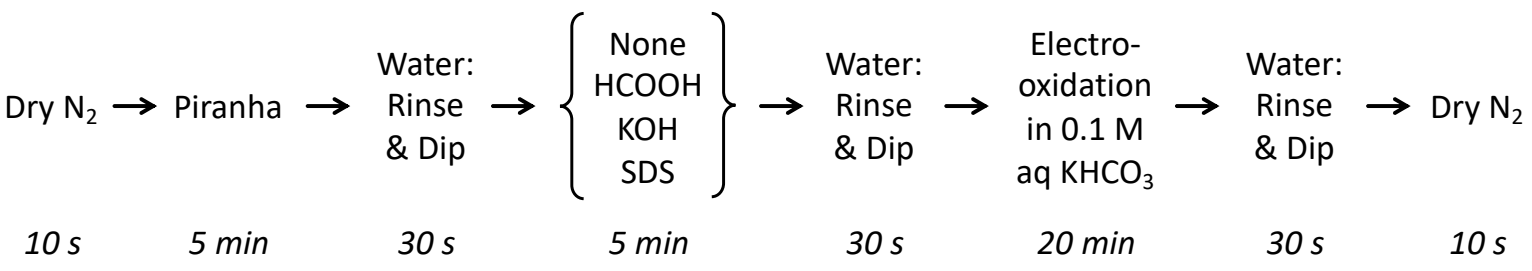

B

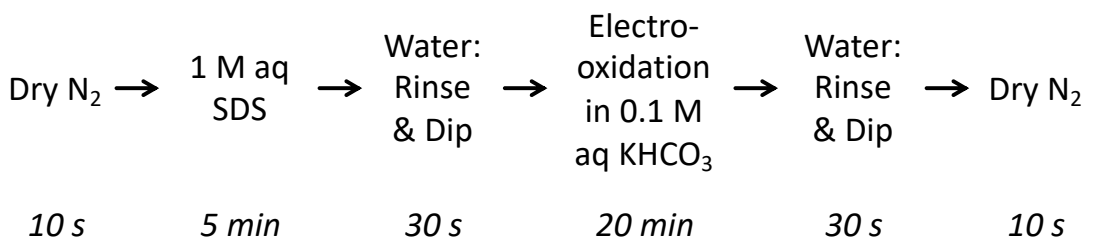

Fig. 3. Chemical treatment protocols for CFP. A Acid-containing processes with systematic iterations of a second chemical etch. B Acid-free, green chemistry process for CFP that takes less than 30 minutes, imparts long-lasting hydrophilicity, and leaves the CFP structurally intact. Times for each step are stated in italic; aq is aqueous.

We found that the acid-free procedure greatly outperformed the other twelve solutionprocessable treatments in terms of sustained hydrophilicity (Fig. 4), paving the way for creating large areas of hydrophilic CFP via a benign, environmentally friendly process, without the need for harsh acids, which significantly improved chemical safety and reduced the generation of hazardous waste. 


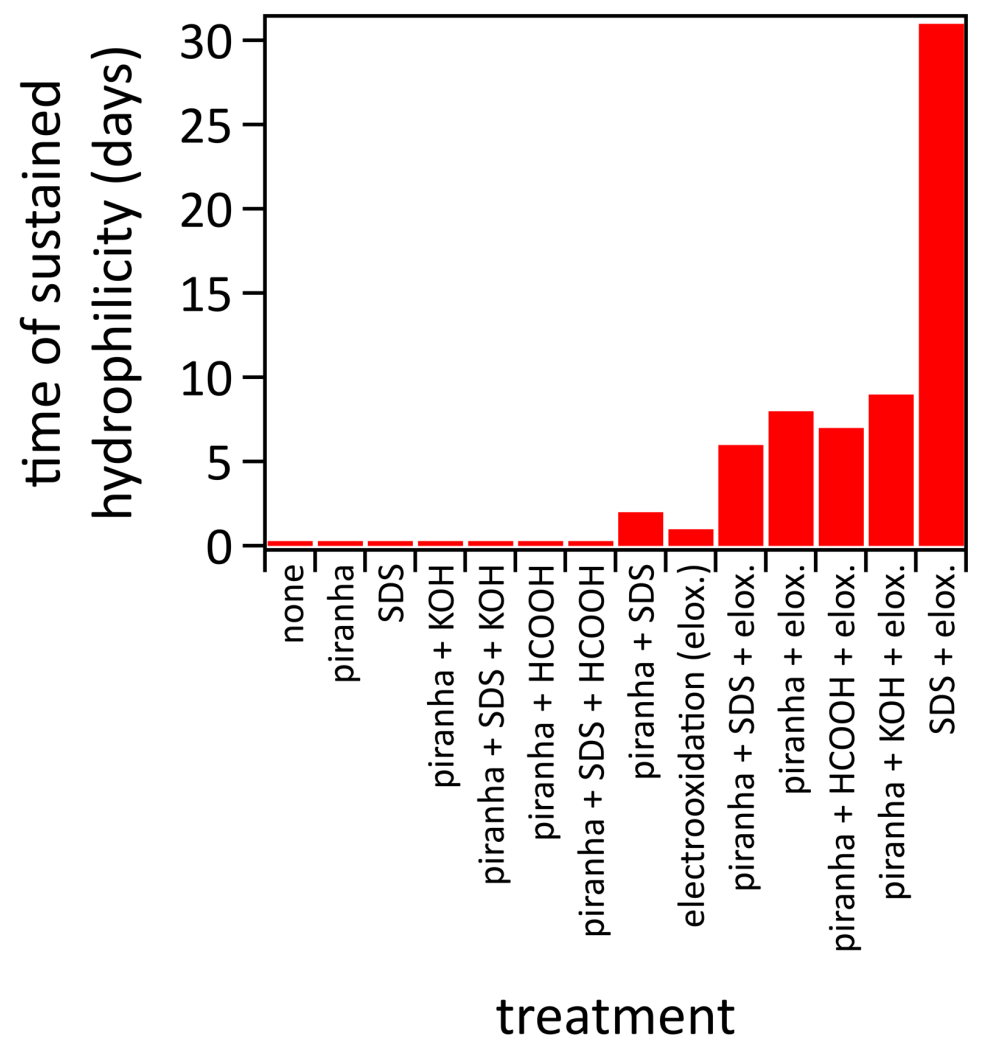

Fig. 4. Durability of hydrophilicity of untreated and treated CFP upon storage in ambient air. Piranha refers to soaking for 5 minutes in a 4:1 (v/v) mixture of concentrated sulfuric acid and $30 \%$ aqueous hydrogen peroxide solution, SDS refers to sonication for 5 minutes in a $1 \mathrm{M}$ aqueous sodium dodecyl sulfate solution, $\mathrm{KOH}$ refers to soaking for 5 minutes in a $6 \mathrm{M}$ aqueous $\mathrm{KOH}$ solution, $\mathrm{HCOOH}$ refers to soaking for 5 minutes in concentrated formic acid, and electrooxidation (elox.) was performed in $0.1 \mathrm{M} \mathrm{pH} 8.7$ aqueous $\mathrm{KHCO}_{3}$ electrolyte at $+1.63 \mathrm{~V}$ vs. $\mathrm{Ag} / \mathrm{AgCl}$ for 20 minutes.

Evaluation of all thirteen chemical treatments revealed that electrooxidation in $0.1 \mathrm{M}$ aqueous $\mathrm{KHCO}_{3}$ electrolyte after chemical etches was critical for sustained hydrophilicity. We deliberately chose a mild aqueous electrolyte, with the aim to retain the structural integrity of CFP. All chemical procedures that did not contain an electrooxidation step, i.e., one-step piranha or SDS treatments, 
or two-step piranha-SDS, piranha-KOH, or piranha-HCOOH treatments, or three-step piranhaSDS-KOH or piranha-SDS-HCOOH treatments, led to CFP that became hydrophobic within only two days (Fig. 4). Hydrophilicity of piranha-SDS treated CFP lasted for two days, while all other chemical procedures that did not contain an electrooxidation step produced CFP that lost hydrophilicity within three hours. However, electrooxidation by itself was not sufficient for sustained hydrophilicity; electrooxidized CFP became hydrophobic after only one day.

Chemical treatments that started with immersion in piranha solution and concluded with electrooxidation generated CFP with hydrophilicities that lasted for about a week (Fig. 4). The two-step piranha-electrooxidation treatment of CFP resulted in sustained hydrophilicity for 8 days. We added a second chemical step after the piranha treatment (Fig. 3A) to better understand what drives durability of hydrophilicity. This second chemical step consisted of soaking the piranha treated and washed CFP in concentrated formic acid or $6 \mathrm{M}$ aqueous $\mathrm{KOH}$ or sonicating it in $1 \mathrm{M}$ aqueous SDS solution. The rationale for choosing these three chemicals was that formic acid may induce carboxylic surface functional groups, $\mathrm{KOH}$ may facilitate the formation of hydroxyl or carboxylic surface functional groups, and the detergent SDS would thoroughly eliminate organic or otherwise labile contaminants from the carbon surface before electrooxidation. Another reason was the wide $\mathrm{pH}$ range that these three chemicals cover. We hypothesized that another acid treatment may further oxidize surface carbon, whereas the strong base would lead to surface reactions complementary to the preceding strong acid treatment, possibly also further oxidizing surface carbon. In contrast, the more dilute $1 \mathrm{M}$ aqueous SDS solution may be advantageous for applications of treated CFP in environments that are sensitive to strong acids or bases, which may be left in small quantities in the porous three-dimensional network of carbon fibers. We note that such residual acids or bases can only be washed away with water if the CFP surface is sufficiently 
hydrophilic for water to fully penetrate into the three-dimensional carbon fiber network. Threestep treatments of CFP, i.e., piranha-SDS-electrooxidation, piranha-HCOOH-electrooxidation or piranha- $\mathrm{KOH}-$ electrooxidation, gave rise to sustained hydrophilicity of 6,7 , or 9 days, respectively. SEM images showed that all thirteen chemical treatments we employed to impart hydrophilicity to CFP left the carbon fibers and their network architectures intact (Fig. 2 and S2), suggesting that our solution-processable carbon surface modifications provided a substantial advantage over previously reported procedures.

Identification of surface species controlling sustained hydrophilicity. We evaluated X-ray photoelectron spectroscopy (XPS) data to show that our acid-free treatment selectively produced surface hydroxyl groups, which in turn were responsible for long-lasting hydrophilicity of CFP. We chose XPS to assess CFP surfaces because XPS is a surface-sensitive technique and well suited to assess oxygenated carbon species, i.e. $\mathrm{C}-\mathrm{O}, \mathrm{C}=\mathrm{O}$ and $\mathrm{O}-\mathrm{C}=\mathrm{O}$ functional groups, on graphitic carbon. ${ }^{32}$ However, small chemical shifts between carboxyl, hydroxyl, and ether groups on carbon $^{41-44}$ resulted in a single $\mathrm{C}-\underline{\mathrm{O}}$ peak for all three surface functional groups. Likewise, $\mathrm{C}=\underline{\mathrm{O}}$ peaks included carboxyls, aldehydes, carbonyls, esters, and ketones, which have similar central binding energies ${ }^{41-44}$ and therefore cannot be differentiated by XPS. Similarly, each C 1s peak was comprised of multiple functional groups as outlined in Table 1. Nevertheless, we were able to disentangle which surface functional groups governed durability of hydrophilicity. 
Table 1. Central binding energy (BE) ranges, surface species, and functional groups of oxygenated carbon species on untreated and treated CFP.

\begin{tabular}{|c|c|c|c|c|c|}
\hline core level & \multicolumn{2}{|c|}{$\mathrm{O} 1 \mathrm{~s}$} & \multicolumn{3}{|c|}{ C 1s } \\
\hline species & $\mathrm{C}=\underline{\mathrm{O}}$ & $\mathrm{C}-\underline{\mathrm{O}}$ & $\underline{\mathrm{C}}-\mathrm{O}$ & $\underline{\mathrm{C}}=\mathrm{O}$ & $\mathrm{O}-\underline{\mathrm{C}}=\mathrm{O}$ \\
\hline $\mathrm{BE}$ range $(\mathrm{eV})$ & $531.6-532.3$ & $533.0-533.7$ & $286.4-287.0$ & $287.4-288.0$ & $288.7-289.2$ \\
\hline \multirow{5}{*}{ functional groups } & carboxyls & carboxyls & hydroxyls & aldehydes & carboxyls \\
\hline & aldehydes & hydroxyls & esters & carbonyls & esters \\
\hline & carbonyls & ethers & ethers & ketones & \\
\hline & esters & & & & \\
\hline & ketones & & & & \\
\hline
\end{tabular}

We collected XPS data on the day of treatment, identified and quantified surface species, and correlated them to the observed time of sustained hydrophilicity of CFP, which was stored in ambient air (Fig. 4). Survey XP spectra revealed that only carbon and oxygen were present at untreated and treated CFP surfaces (Fig. S3). We note that peak-fitting and assignment of C 1s species on graphitic carbon substrates is not trivial. ${ }^{45}$ High-resolution $\mathrm{C}$ 1s spectra required six peaks to match the measured data (Fig. S4 and S5). Our C 1s spectra exhibited asymmetric shape and shake-up features, as observed before for graphitized $\mathrm{sp}^{2}$-like carbon. ${ }^{46-49}$ The central binding energy of graphitic carbon ranged from 284.5 to $285.0 \mathrm{eV}$, in agreement with reported values..$^{50,51}$ Additionally, adventitious carbon was present, whose central binding energy was taken to be at $284.8 \mathrm{eV} .{ }^{52}$ We note that globally fixing the adventitious to graphitic carbon ratio did not change 
carbon or oxygen content results; therefore, we did not fix this ratio because the amount of adventitious carbon on each sample is unknown. We normalized high-resolution $\mathrm{C}$ 1s and $\mathrm{O}$ 1s peaks of oxygenated carbon species to the sum of graphitic and adventitious carbon species to account for overall signal strength differences from sample to sample; relative sensitivity factors resulting from photoemission cross-sections and analyzer transmission of photoelectrons were also considered.

We detected oxygenated carbon species in the high-resolution $\mathrm{C} 1 \mathrm{~s}$ and $\mathrm{O} 1 \mathrm{~s}$ spectra and made assignments based on both elemental regions (Table 1). The O 1s spectra required two peaks to match the experimental data (Fig. S6). These peaks had central binding energies of 531.6 to 532.3 $\mathrm{eV}$, attributable to $\mathrm{C}=\underline{\mathrm{O}}$ functional groups, such as in carboxyls, aldehydes, carbonyls, esters, and ketones, and 533.0 to $533.7 \mathrm{eV}$, attributable to $\mathrm{C}-\underline{\mathrm{O}}$ species, such as in carboxyls, hydroxyls, and ethers. ${ }^{41-44}$ Accordingly, we constrained the central binding energies of the remaining three peaks in the $\mathrm{C}$ 1s spectra to the following ranges: 286.4 to $287.0 \mathrm{eV}$ ( $\underline{\mathrm{C}}-\mathrm{O}$; hydroxyls, esters, and ethers), 287.4 to $288.0 \mathrm{eV}(\underline{\mathrm{C}}=\mathrm{O}$; aldehydes, carbonyls, and ketones), and 288.7 to $289.2 \mathrm{eV}(\mathrm{O}-\underline{\mathrm{C}}=\mathrm{O}$; carboxyls and esters). ${ }^{41-44,53-55}$ Our multi-peak fits matched the measured data well (Fig. S4 to S6) and provided quantifications of individual oxygenated carbon species.

High-resolution XP spectra in the $\mathrm{C} 1 \mathrm{~s}$ and $\mathrm{O}$ 1s core level regions showed significant changes of surface characteristics between untreated and treated CFP (Fig. 5 and S4 to S6). Untreated, hydrophobic CFP exhibited only $0.85 \%$ total surface oxygen relative to the sum of graphitic and adventitious surface carbon. In contrast, SDS-electrooxidation treated CFP, whose hydrophilicity lasted for 31 days, had 10 times more total surface oxygen (8.5\%) on the day of preparation (Fig. 5 and S4). 


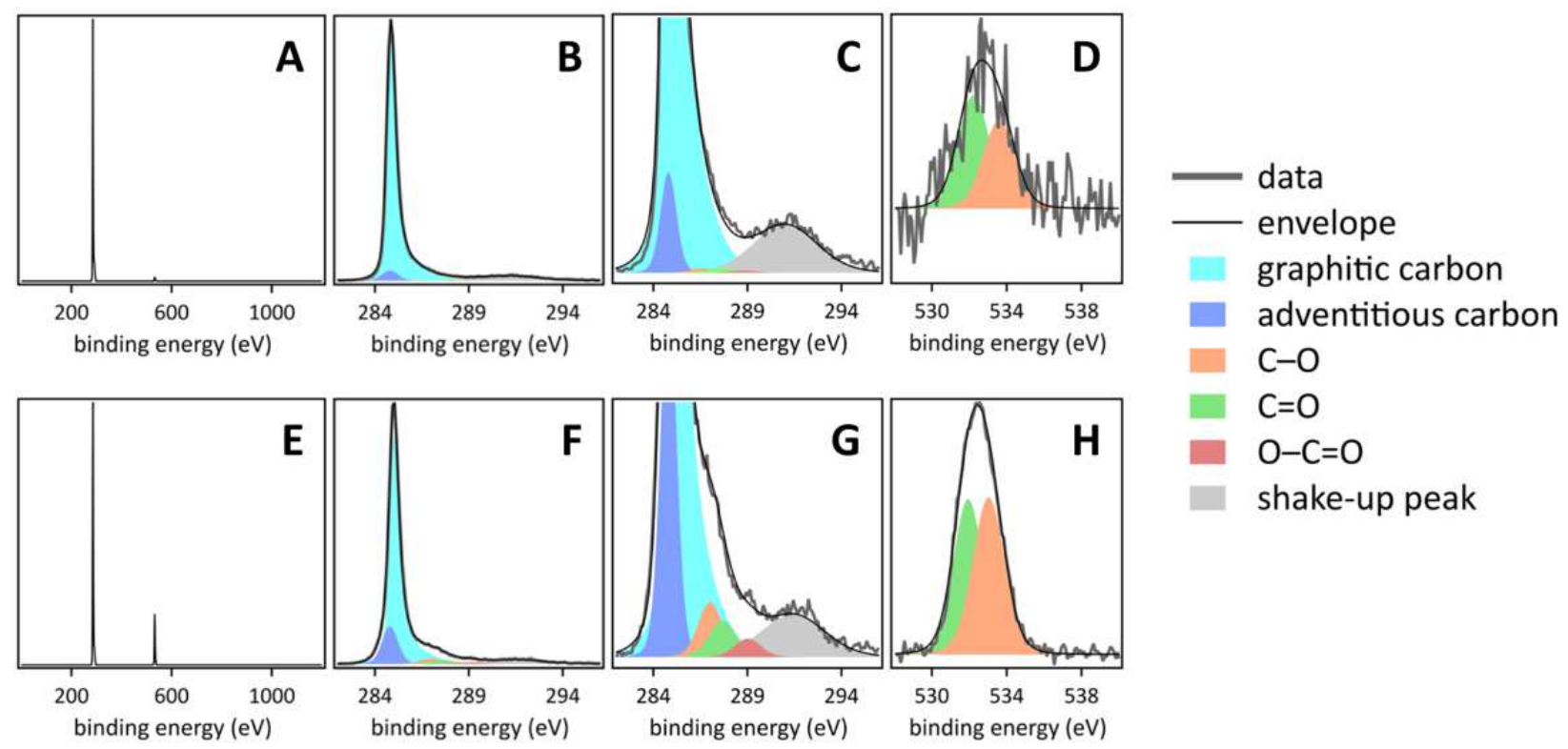

Fig. 5. XPS data and peak fits of untreated and green chemistry processed CFP. A, B, C, D Untreated CFP. E, F, G, H SDS-electrooxidation treated CFP. A, E Survey XP spectra. B, F Highresolution $\mathrm{C} 1 \mathrm{~s}$ spectra. $\mathbf{C}, \mathbf{G}$ High-resolution $\mathrm{C}$ 1s spectra, with a $y$-axis that was magnified by a factor of 10. D, H High-resolution O 1s spectra.

We found that oxygen content in $\mathrm{C}-\underline{\mathrm{O}}$ species, generally attributable to carboxyls, hydroxyls, and ethers, ${ }^{41-44}$ correlated best with the observed trends in durability of hydrophilicity (Fig. 4 and S7A). All CFP samples that were hydrophilic for ca. a week or longer possessed greater than $2.3 \%$ surface $\mathrm{C}-\underline{\mathrm{O}}$ content, whereas $\mathrm{CFP}$ that became hydrophobic within two days had surface $\mathrm{C}-\underline{\mathrm{O}}$ contents below 1.7\% (Fig. S7A).

The nature of the chemical treatment did matter for the observed surface $\mathrm{C}-\underline{\mathrm{O}}$ content of CFP. Strikingly, treatment in $1 \mathrm{M}$ aqueous SDS led to higher surface $\mathrm{C}-\underline{\mathrm{O}}$ content than immersion in harsh piranha acid; however, hydrophilicity lasted only 3 hours for both preparations (Fig. 4). Treatments without electrooxidation led to hydrophilicity that lasted up to only two days, and corresponding surface $\mathrm{C}-\underline{\mathrm{O}}$ content remained below 1.7\% (Fig. S7A). Likewise, electrooxidation 
alone produced hydrophilicity that lasted only one day, and the surface $\mathrm{C}-\underline{\mathrm{O}}$ content was $0.7 \%$. CFP treated with piranha solution and electrooxidation, plus possibly a second chemical etch, maintained hydrophilicity for about a week (Fig. 4), and corresponding surface $\mathrm{C}-\underline{\mathrm{O}}$ contents ranged from 2.4 to $4.4 \%$ (Fig. S7A). Our acid-free procedure, which imparted sustained hydrophilicity for 31 days upon storage in ambient air, led to 4.4\% surface C- $\underline{\mathrm{O}}$ content. Overall, surface $\mathrm{C}-\underline{\mathrm{O}}$ content derived from XPS data taken on the day of sample preparation was predictive of durability of hydrophilicity.

Aprotic oxygenated functional groups, i.e., carbonyls, esters, ethers, and ketones, induce surface dipole moments but lack hydrogen that is needed to form strong hydrogen bonding networks to water molecules at the solid-liquid interface. Thus, such aprotic groups contribute less to sustained hydrophilicity than protic oxygenated functional groups, i.e., carboxyls, aldehydes, and hydroxyls. Since surface $\mathrm{C}-\underline{\mathrm{O}}$ content was predictive of the observed durability of hydrophilicity (Fig. 4 and S7A), and only protic functional groups enable strong hydrogen bonding networks at the carbonwater interface, we conclude that the $\mathrm{C}-\underline{\mathrm{O}}$ peaks, and as a consequence all CFP surfaces, did not contain considerable amounts of ethers. Likewise, surface $\underline{\mathrm{C}}-\mathrm{O}$ content was indicative of sustained hydrophilicity (Fig. 4 and S7C), suggesting that the amounts of aprotic esters that were present on CFP surfaces were insignificant. Surface $\mathrm{O}-\underline{\mathrm{C}}=\mathrm{O}$ content arose from carboxyls and esters; surface amounts of esters were likely unimportant to overall signal strengths as deduced from surface $\underline{\mathrm{C}}-$ O content data. Surface $\mathrm{O}-\underline{\mathrm{C}}=\mathrm{O}$ contents were overall low and did not correlate with the durability of hydrophilicity data (Fig. 4 and S7E), implying that surface carboxyls were not necessary for long-lasting CFP hydrophilicity. Linking this result together with our observation that surface C-

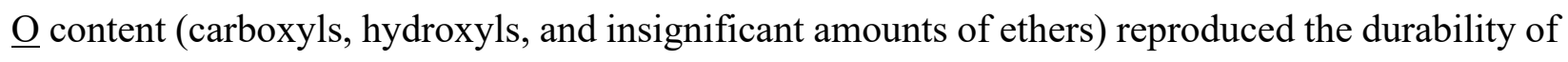
hydrophilicity trends well allowed us to conclude that surface hydroxyls, not carboxyls, controlled 
the durability of hydrophilicity. Further, we found that the surface $\mathrm{O}-\underline{\mathrm{C}}=\mathrm{O}$ content, indicative of carboxyls, was higher for piranha-SDS-electrooxidation treated CFP $(1.7 \% ; 6$ days of sustained hydrophilicity) than for SDS-electrooxidation treated CFP (1.4\%; 31 days of sustained hydrophilicity), which corroborates that carboxyls were not predictive of long-lasting hydrophilicity.

Finally, all XPS data taken together indicate that the $\mathrm{C}-\underline{\mathrm{O}}$ signals arose from surface hydroxyls. Surface $\mathrm{C}-\underline{\mathrm{O}}$ species are comprised of carboxyls, hydroxyls, and ethers (Table 1), but we ruled out prevalent roles of carboxyls and ethers. That means that greater than $2.3 \%$ surface hydroxyl content, normalized to total non-oxidized surface carbon, was required for sustained hydrophilicity for ca. one week or longer.

Interpretation of XPS signals arising from $\mathrm{C}=\underline{\mathrm{O}}$ (carboxyls, aldehydes, carbonyls, esters, and ketones) and $\underline{\mathrm{C}}=\mathrm{O}$ (aldehydes, carbonyls, and ketones) is less straightforward, but may be useful in explaining our observation of equally high surface $\mathrm{C}-\underline{\mathrm{O}}$ contents of $4.4 \%$ in piranha-SDSelectrooxidation treated vs. SDS-electrooxidation treated CFP, although these treatments led to markedly different durability of hydrophilicity of 6 vs. 31 days, respectively. Surface $\mathrm{C}=\underline{\mathrm{O}}$ and $\underline{\mathrm{C}}=\mathrm{O}$ contents did not correlate well with the observed hydrophilicity data (Fig. 4 and S7B and S7D), indicating that non-negligible yet unknown amounts of surface carbonyls and ketones were produced by the chemical treatments; we reiterate that these aprotic oxygenated functional groups increase the wettability of carbon by water less than protic surface groups. Nevertheless, we obtained the highest surface $\mathrm{C}=\underline{\mathrm{O}}$ content of $4.1 \%$ in $\mathrm{SDS}-\mathrm{electrooxidation}$ treated $\mathrm{CFP}$, whereas CFP treated with piranha solution and electrooxidation, plus possibly a second chemical etch, exhibited surface $\mathrm{C}=\underline{\mathrm{O}}$ contents ranging from 2.5 to $3.3 \%$ (Fig. S7B), with piranha-SDSelectrooxidation treated CFP exhibiting 3.2\%. Ergo, we surmise that surface aldehydes may also 
have contributed to sustained hydrophilicity, albeit to a lesser extent than hydroxyls. Overall, our high-resolution XPS data and detailed analysis show that higher amounts of surface hydroxyls on graphitic carbon directly correlated with longer-lasting hydrophilicity of chemically treated CFP. Higher amounts of surface aldehydes aided durability of hydrophilicity of carbon surfaces that exhibited virtually identical high amounts of surface hydroxyls.

Identification of surface hydroxyls as being most beneficial for sustained hydrophilicity explains why previously reported, harsher treatments with strong acids or high temperatures did not generate CFP with long-lived hydrophilicity. More potent oxidants are capable of oxidizing carbon all the way to carboxyls, whereas our mild, acid-free SDS-electrooxidation treatment prevented this detrimental overoxidation and resulted in the selective production of less oxidized surface hydroxyl groups. We note that inhibition of overoxidation of carbon is notoriously challenging because the first oxidation step is thermodynamically most difficult and subsequent oxidations are much easier. Our development of a CFP treatment that provides selectivity for less oxidized hydroxyls over carboxyls greatly enhances the utility of carbon materials and CFP.

Time evolution of surface hydroxyls. Detection of higher amounts of hydroxyl groups on carbon surfaces, i.e. $\mathrm{C}-\underline{\mathrm{O}}$ signals, measured on the day of preparation, was predictive of the durability of hydrophilicity. Therefore, we wanted to know how surface $\mathrm{C}-\underline{\mathrm{O}}$ content evolved over time. Weekly taken XPS data of CFP stored in ambient air whose hydrophilicity lasted for 31 days upon SDS-electrooxidation treatment or 6 days upon piranha-SDS-electrooxidation treatment show that surface $\mathrm{C}-\underline{\mathrm{O}}$ content diminished over time but remained above the critical threshold of 2.3\% as long as CFP was hydrophilic (Fig. 6A). The surface C- - O content of SDS-electrooxidation treated CFP, which remained hydrophilic for 31 days in ambient air, started at $4.4 \%$ and decayed 
to $2.2 \%$ after five weeks, when SDS-electrooxidation treated CFP eventually became hydrophobic (Fig. 6A).
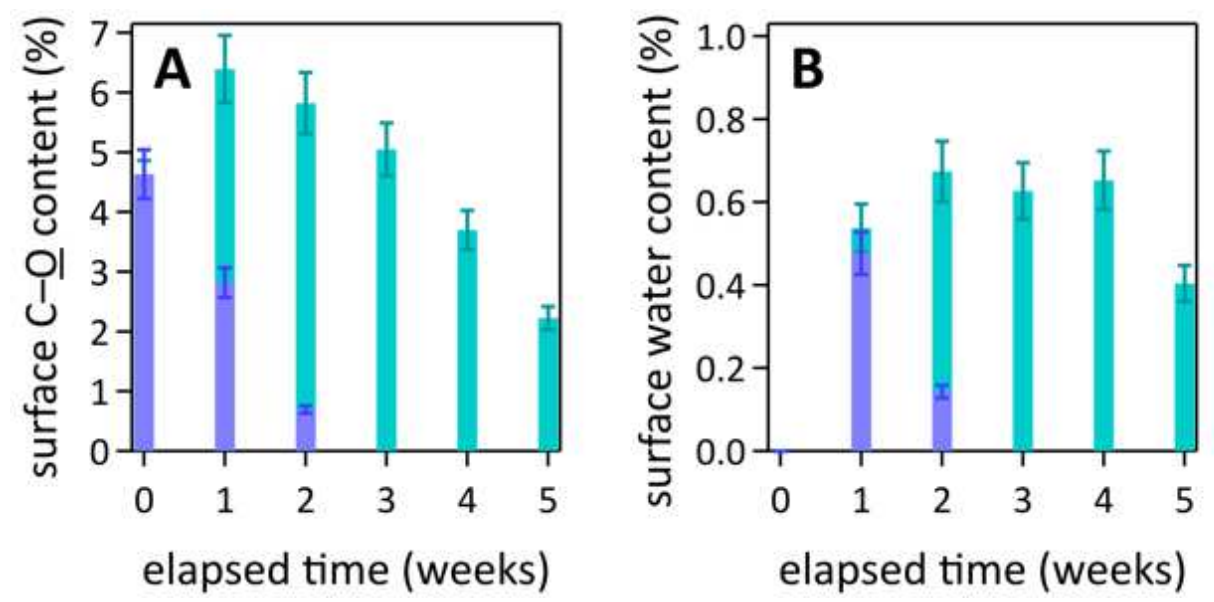

Fig. 6. Time evolution of surface hydroxyls and adsorbed water. A Surface $\mathrm{C}-\underline{\mathrm{O}}$ content as a function of weeks elapsed between treatment and XPS data collection. B Surface content of adsorbed water as a function of time. Cyan, SDS-electrooxidation treated CFP; purple, piranhaSDS-electrooxidation treated CFP. Samples were stored in ambient air.

Interestingly, the surface $\mathrm{C}-\underline{\mathrm{O}}$ content increased after one week of storage in ambient air to $6.4 \%$, after which it steadily decreased. We surmise that adsorption of water from ambient air, which had an average relative humidity of ca. $78 \%$, on hydrophilic carbon fibers led to surface reactions that produced species that contributed to the surface $\mathrm{C}-\underline{\mathrm{O}}$ content. We found that after storage for a week or more in ambient air we had to include a third peak with a central binding energy ranging from 535.3 to $536.0 \mathrm{eV}$, attributable to adsorbed water, ${ }^{45,56}$ to match the $\mathrm{O} 1 \mathrm{~s}$ signal (Fig. S8). Adsorbed water has been observed before on carbon surfaces, with central binding energies of 535.3 to $536.1 \mathrm{eV} .{ }^{45,56}$ The presence of adsorbed water and its interaction with hydrophilic surface carbon complicated the interpretation of the evolution of surface $\mathrm{C}-\underline{\mathrm{O}}$ content over time and its correlation to wettability of CFP by water. Quantification of adsorbed water showed that the 
surface content of chemisorbed water was ca. 0.6 to $0.7 \%$ upon storage of SDS-electrooxidation treated CFP for one to four weeks in ambient air, while the CFP was still hydrophilic, and decreased to $0.4 \%$ after hydrophilicity was lost (Fig. 6B). For comparison, analogous data for piranha-SDS-electrooxidation treated CFP, which remained hydrophilic for only 6 days in ambient air, are also depicted in Fig. 6. The surface $\mathrm{C}-\underline{\mathrm{O}}$ content of piranha-SDS-electrooxidation treated CFP decreased steadily with time. We note that piranha-SDS-electrooxidation treated CFP was already hydrophobic after one week of storage in ambient air and its surface content of adsorbed water was $0.5 \%$, which decayed to $0.1 \%$ after two weeks in ambient air, suggesting that water chemisorption was more prevalent on hydrophilic carbon surfaces. Elemental analysis by SEM-EDX data provided evidence that only carbon and oxygen were present after storage of CFP in ambient air (Fig. S9). SEM images showed that storage in ambient air did not structurally damage the carbon fiber network architectures (Fig. S10).

Purity of treated carbon surfaces. We collected XPS data in the Na 1s, S 2p, or K 2s corelevel regions (Fig. S11) to exclude the presence of surface residues from the applied chemical treatments. We chose these high-resolution regions because the chemical treatments we employed contained the respective chemical elements. For example, sulfuric acid in piranha solution could be a source of surface sulfur, SDS could give rise to surface sodium and sulfur, and electrooxidation in aqueous $\mathrm{KHCO}_{3}$ electrolyte could leave surface potassium. We chose to inspect the $\mathrm{K} 2 \mathrm{~s}$ instead of the $\mathrm{K} 2 \mathrm{p}$ core-level region because the $\mathrm{K} 2 \mathrm{p}$ and $\mathrm{C} 1 \mathrm{~s}$ signals overlap. Additionally, we monitored the Fe $2 \mathrm{p}$ core-level region for surface iron because iron is ubiquitous in glassware and many chemicals ${ }^{57}$ None of these elements, other than carbon and oxygen, were present at CFP surfaces (Fig. S11). Elemental analysis by SEM-EDX spectroscopy corroborated that CFP surfaces contained only carbon and oxygen (Fig. S12). 
Preservation of hydrophilicity by storage under water. For practical applications of CFP, hydrophilicity must be sustained during operation in water or aqueous media, and not necessarily with intermittent storage in ambient air. Therefore, we conducted long-term time trials of select preparations of CFP that were stored under deionized water. We tested CFP that was treated by our acid-free SDS-electrooxidation or acid-containing piranha-SDS-electrooxidation procedures. For better comparability, we prepared ten separate samples for each treatment to be tested for the first 10 weeks and kept all of them under deionized water from day one, so that each sample was dried only once at the time of assessment. After 10 weeks, we dried and re-immersed the samples that had been under water for 10 weeks already and further re-tested their hydrophilicity every 7 days. Hydrophilicity of SDS-electrooxidation and piranha-SDS-electrooxidation treated CFP lasted for more than 5 months upon storage under water. Elemental analysis by SEM-EDX data provided evidence that only carbon and oxygen were present after long-term storage of CFP in deionized water (Fig. S13). SEM images showed that storage under water did not structurally damage the carbon fiber network architectures (Fig. S14), rendering our acid-free procedure to impart long-lasting hydrophilicity to CFP transformative for many applications, including in clean energy technologies, such as aqueous electrolyzers, flow batteries, and supercapacitors, as well as water purification, electronics, biomedical, and sensing, especially biosensing, applications.

\section{Conclusions}

Selective carbon surface hydroxylation of initially hydrophobic CFP by an acid-free, environmentally benign, solution-processable method, comprised of treatment in $1 \mathrm{M}$ aqueous SDS solution, followed by electrooxidation in $0.1 \mathrm{M}$ aqueous bicarbonate electrolyte, imparted sustained hydrophilicity for 31 days when CFP was stored in ambient air; CFP remained 
hydrophilic for more than 5 months when stored under water. The procedure takes less than 30 minutes of preparation time and is amenable to large-scale manufacturing. Importantly, the treatment did not embrittle CFP, as evidenced by the excellent bendability of treated CFP. SEM images showed that the carbon fibers and their network architectures remained intact, suggesting that our green chemistry carbon surface modification provided a substantial advantage over previously reported procedures.

Systematic iterations of thirteen different chemical treatments together with XPS and durability of hydrophilicity data enabled the identification of hydroxyl, not carboxyl, surface functional groups to control the durability of hydrophilicity. Our acid-free carbon surface modification treatment provided selectivity for less oxidized hydroxyls over carboxyls and transforms the utility of carbon materials. Inhibition of overoxidation of carbon beyond hydroxylation was achieved by the mild treatment conditions. SEM-EDX and XPS data showed that the chemical treatments did not leave residues, as only carbon and oxygen were detected on CFP surfaces. Overall, our results highlight the exceptional utility of SDS-electrooxidation treated CFP in clean energy, water purification, electronics, sensing, and biomedical applications.

\section{Methods}

All chemicals were used as received. Deionized water was obtained from a Thermo Scientific Barnstead Smart2Pure Pro UV/UF 15 LPH Water Purification System and had a resistivity of $\geq 17.5 \mathrm{M} \Omega \cdot \mathrm{cm}$. All experiments were performed at room temperature. All dry nitrogen gas came from oil-free liquid nitrogen boil-off to prevent transfer of compression oil, which most compressed gases contain, onto the carbon fiber paper (CFP). Data analysis and graphing were performed with Igor Pro 8.04 (Wavemetrics). 
CFP surface treatment. Uncoated, initially hydrophobic CFP (AvCarb MGL190, FuelCellStore) was cut into pieces that were ca. $23 \mathrm{~mm}$ wide and $39 \mathrm{~mm}$ high, called henceforward rectangular part. They also exhibited a tab for electrical contacting in an electrochemical cell on one short side that was ca. $8 \mathrm{~mm}$ wide and $15 \mathrm{~mm}$ high, called henceforward tab (Fig. 2C). A few samples were strips with ca. $1.5 \mathrm{~cm}$ width and ca. $4.0 \mathrm{~cm}$ height. Clean plastic tweezers were used to handle CFP to prevent metal contaminations. Each entire CFP piece was first held for ca. $10 \mathrm{~s}$ in a dry nitrogen stream to remove loose debris. Acid-free treatment consisted of sonication of the rectangular part of CFP for 5 minutes in a $1.0 \mathrm{M}$ aqueous sodium dodecyl sulfate (SDS, AG Scientific, $\geq 99 \%$ ) solution; a 1210 Branson bath sonicator was used, it delivered 80 watts. After that the rectangular part was subjected to electrooxidation in $0.1 \mathrm{M}$ aqueous potassium bicarbonate $\left(\mathrm{KHCO}_{3}\right.$, Alfa Aesar, 99.7-100.5\%) electrolyte in a standard three-electrode cell; the $\mathrm{pH}$ was 8.72, as determined by a Thermo Scientific Accumet Excel XL20 pH meter. The pretreated CFP piece served as working electrode. The counter electrode was also CFP to prevent metal contaminations, and the distance between working and counter electrode was ca. $15 \mathrm{~mm}$. The reference electrode was $\mathrm{Ag} / \mathrm{AgCl}(3 \mathrm{M}$ aqueous $\mathrm{NaCl}, \mathrm{BASi})$. The potential was set to $+1.63 \mathrm{~V}$ vs. $\mathrm{Ag} / \mathrm{AgCl}$ for 20 minutes. We optimized the electrooxidation time in our acid-free treatment with respect to durability of hydrophilicity and time of the procedure. We used CFP, which had been sonicated in $1 \mathrm{M}$ aqueous SDS solution, and varied the electrochemical treatment time from 5 to 60 minutes. We assessed the time samples sank in deionized water without agitation. We found that electrooxidation times longer than 20 minutes did not improve the durability of hydrophilicity (Fig. S15). Therefore, we chose 20 minutes electrooxidation time throughout our experiments. After electrooxidation, the entire CFP piece was first rinsed with and then dipped in deionized water for ca. 30 seconds. Finally, the entire CFP piece was dried in a dry nitrogen stream. 
Piranha treatment consisted of immersion of the rectangular part for 5 minutes in a 4:1 (v/v) mixture of concentrated sulfuric acid (Fisher, ACS Plus) and 30\% aqueous hydrogen peroxide solution (Fisher), after the initial dry nitrogen stream exposure. After this piranha treatment, the entire CFP piece was first rinsed with and then dipped for ca. $30 \mathrm{~s}$ in deionized water. Excess water was shaken off mechanically. On some samples a second chemical etch was employed after the piranha treatment: the rectangular part was immersed and sonicated for 5 minutes in a $1.0 \mathrm{M}$ aqueous SDS solution. Or the second etch consisted of soaking for 5 minutes in concentrated formic acid (J. T. Baker, $\geq 90 \%$ ) or in $6.0 \mathrm{M}$ aqueous $\mathrm{KOH}$ (Sigma-Aldrich, $\geq 99.99 \%$ ). After that, the entire CFP piece was first rinsed with and then dipped for ca. $30 \mathrm{~s}$ in deionized water. Then, the entire carbon fiber paper piece was dried in a dry nitrogen stream, to prevent the transfer of additional water into the next step. Some samples that were treated with this second chemical etch were subsequently electrooxidized, using the conditions described above. Select CFP samples were thermally treated in ambient air at 500 or $800{ }^{\circ} \mathrm{C}$, using ceramic boats and a Thermolyne Model 48000 furnace vented to an exhaust.

We prepared several triplicates of a preparation that consisted of soaking in piranha solution, sonication in $1 \mathrm{M}$ aqueous SDS solution, followed by electrooxidation, using the conditions described above, and found excellent reproducibility. Samples were stored in ambient air or under deionized water. The average relative humidity of ambient air was ca. $78 \%$ during summer in Rochester, New York.

Long-term time trials to assess the durability of hydrophilicity upon storage under deionized water were performed with CFP treated by acid-free SDS-electrooxidation or acid-containing piranha-SDS-electrooxidation procedures. Ten samples of each preparation were made and immediately stored under deionized water in new Falcon tubes at room temperature. 
Hydrophilicity was assessed every 7 days up to 10 weeks via the sink-or-float test after drying in a dry nitrogen stream. Pieces of that CFP sample were cut off for physical characterization; samples were discarded after a particular week's testing. After 10 weeks, we re-immersed the samples that had been under water for 10 weeks already and further re-tested their hydrophilicity every 7 days.

Hydrophilicity assessment. We tested for hydrophilicity of CFP surfaces by the sink-or-float test in deionized water (Fig. S16). Dry CFP pieces were dropped from ca. $5 \mathrm{~cm}$ above the water surface into a beaker halfway filled with deionized water. When dry CFP is dropped into water, hydrophilic CFP immediately sinks because hydrophilic carbon surfaces are sufficiently wetted by water to displace all air trapped in dry CFP. In contrast, hydrophobic CFP floats because its carbon surfaces repel water so that trapped air cannot escape, rendering hydrophobic CFP buoyant. Visual inspection detected sinking or floating. Pieces that floated were additionally agitated with plastic tweezers and pushed under water to monitor sinking with agitation or resurfacing (Fig. S16). Time of sustained hydrophilicity refers to the time passed until a CFP piece stopped sinking altogether, even upon agitation.

Physical characterization. Scanning electron microscopy (SEM) images were obtained using a JEOL JSM-5900LV SEM instrument equipped with a thermionic tungsten electron gun, operated at $25 \mathrm{kV}$ with a working distance of $10 \mathrm{~mm}$. CFP samples were mounted on 1-inch diameter aluminum SEM stubs (Ted Pella) with carbon tape (Electron Microscopy Sciences). A liquidnitrogen cooled EDAX CDU LEAP detector was used in conjunction with the EDAX Genesis software package.

X-ray photoelectron spectra (XPS) were collected using a Kratos Axis Ultra XPS instrument equipped with a monochromatized $\mathrm{Al} \mathrm{K} \alpha$ radiation source, operated in high-power mode at 200 
$\mathrm{W}$ and $15 \mathrm{kV}$, with a base chamber pressure of $3.0 \times 10^{-8}$ mbar. Samples were mounted on doublesided adhesive copper tape. Survey scans were obtained between 0 and $1200 \mathrm{eV}$ with a step size of $1 \mathrm{eV}$, a dwell time of $200 \mathrm{~ms}$, and an analyzer pass energy of $140 \mathrm{eV}$ averaged over 5 scans. Core-level region scans for $\mathrm{C} 1 \mathrm{~s}, \mathrm{O} 1 \mathrm{~s}, \mathrm{Na} 1 \mathrm{~s}, \mathrm{~S} 2 \mathrm{p}, \mathrm{K} 2 \mathrm{~s}$, and Fe 2p were obtained at the corresponding binding energy ranges with a step size of $0.1 \mathrm{eV}$, an average dwell time of $260 \mathrm{~ms}$, and an analyzer pass energy of $20 \mathrm{eV}$ averaged over 5 scans. Binding energies were referenced to the $\mathrm{C}$ 1s peak arising from adventitious carbon, taken to have a binding energy of $284.8 \mathrm{eV} .{ }^{52}$ Binding energies and quantitative peak areas were derived after Shirley background subtraction ${ }^{58}$ and Gaussian/Lorentzian envelope peak fitting. For the quantification of different components, instrument-specific atomic sensitivity factors determined from standard materials were used. Quantitative XPS analysis was performed with CasaXPS (Version 2.3.24).

\begin{abstract}
Abbreviations
aq; aqueous; BE, binding energy; CFP, carbon fiber paper; EDX, energy-dispersive X-ray; elox., electrooxidation; SDS, sodium dodecyl sulfate; SEM, scanning electron microscopy; v, volume; XP, X-ray photoelectron; XPS, X-ray photoelectron spectroscopy.
\end{abstract}

\title{
Data availability
}

SEM images, SEM-EDX data, XPS data and peak fits, data for optimization of electrooxidation time, and photographs of sink-or-float testing are available in the Supplementary Information. All data are available from the authors upon request. 


\section{Acknowledgments}

K.R.W. and O.C.F. are thankful for funding through Eisenberg Summer Research Fellowships awarded by the Department of Chemical Engineering at the University of Rochester. B.P.Y. acknowledges support for summer research by a Discover Grant of the University of Rochester. Generous financial support from the University of Rochester through start-up funds is acknowledged.

\section{Author contributions}

A.M.M. conceived and supervised the project. M.K.W., K.R.W., O.C.F., and B.P.Y. started the project, prepared treated CFP, optimized conditions, and assessed durability of hydrophilicity. XPS data were collected by M.K.W. and P.R.R., analyzed by M.K.W., and interpreted by M.K.W. and A.M.M. SEM images and SEM-EDX data were acquired and analyzed by C.P.C. The manuscript and the SI were prepared by A.M.M., M.K.W., and C.P.C.

\section{Funding}

Open access funding was provided by the University of Rochester.

\section{Competing interests}

The authors declare no competing financial interest. A patent application has been filed by the University of Rochester with A.M.M., M.K.W., K.R.W., O.C.F., B.P.Y., and C.P.C. as inventors.

\section{Additional information}

Supplementary information The online version contains supplementary material available at $<$ link $>$

Correspondence and requests for materials should be addressed to Astrid M. Müller. 


\section{References}

1. Sun X, Wang Z. Understanding of the Role of Carbon Fiber Paper in Proton Exchange Membrane Fuel Cells. J Electrochem Energy Convers Storage 19, 014501 (2021).

2. Andrews J, Doddathimmaiah AK. 9 - Regenerative fuel cells. In: Materials for Fuel Cells (ed Gasik M). Woodhead Publishing (2008).

3. Wang X, Li W, Xiong D, Petrovykh DY, Liu L. Bifunctional Nickel Phosphide Nanocatalysts Supported on Carbon Fiber Paper for Highly Efficient and Stable Overall Water Splitting. Adv Funct Mater 26, 4067-4077 (2016).

4. Thangavel P, Kim G, Kim KS. Electrochemical integration of amorphous NiFe (oxy)hydroxides on surface-activated carbon fibers for high-efficiency oxygen evolution in alkaline anion exchange membrane water electrolysis. J Mater Chem A 9, 14043-14051 (2021).

5. He H, Lian J, Chen C, Xiong Q, Zhang M. Super hydrophilic carbon fiber film for freestanding and flexible cathodes of zinc-ion hybrid supercapacitors. Chem Eng $J \mathbf{4 2 1}$, 129786 (2021).

6. Tan S, Li J, Zhou L, Chen P, Xu Z. Hydrophilic carbon fiber paper based electrode coated with graphene for high performance supercapacitors. Mater Lett 233, 278-281 (2018).

7. Tan S, Li J, Zhou L, Chen P, Shi J, Xu Z. Modified carbon fiber paper-based electrodes wrapped by conducting polymers with enhanced electrochemical performance for supercapacitors. Polymers 10, 1072 (2018).

8. Hua M, Chen T, Ma H. Design of an intermediate carbon layer between bimetallic sulfide and a carbon-based substrate for high-performance asymmetric supercapacitors. New J Chem $\mathbf{4 2}$, 12511-12519 (2018).

9. Iamprasertkun P, Krittayavathananon A, Sawangphruk M. N-doped reduced graphene oxide aerogel coated on carboxyl-modified carbon fiber paper for high-performance ionic-liquid supercapacitors. Carbon 102, 455-461 (2016).

10. Kaewsongpol T, et al. High-performance supercapacitor of electrodeposited porous 3D polyaniline nanorods on functionalized carbon fiber paper: Effects of hydrophobic and hydrophilic surfaces of conductive carbon paper substrates. Mater Today Commun 4, 176-185 (2015).

11. Huang Z, Ji Z, Yin P, Shu Y, Xu Q, Hu X-Y. Salicylic acid impregnated activated carbon fiber paper: An effective platform for the simple and sensitive detection of hydroxyl radicals in the atmosphere. Electrochem Commun 100, 113-116 (2019). 
12. Yang H, et al. Hierarchical bi-continuous Pt decorated nanoporous Au-Sn alloy on carbon fiber paper for ascorbic acid, dopamine and uric acid simultaneous sensing. Biosens Bioelectron 124-125, 191-198 (2019).

13. Kong W, Wang G, Zhang M, Duan X, Hu J, Duan X. Villiform carbon fiber paper as current collector for capacitive deionization devices with high areal electrosorption capacity. Desalination 459, 1-9 (2019).

14. Coelho IF, et al. Nickel nanoparticles supported by commercial carbon paper as a catalyst for urea electro-oxidation. Mater Renew Sustain Energy 9, 1-11 (2020).

15. Xu S, Jiang Q. Surface modification of carbon fiber support by ferrous oxalate for biofilm wastewater treatment system. J Cleaner Prod 194, 416-424 (2018).

16. Ponchon JL, Cespuglio R, Gonon F, Jouvet M, Pujol JF. Normal pulse polarography with carbon fiber electrodes for in vitro and in vivo determination of catecholamines. Anal Chem 51, 1483-1486 (1979).

17. Kakhki RM. A review to recent developments in modification of carbon fiber electrodes. Arab J Chem 12, 1783-1794 (2019).

18. Torrinha Á, Morais S. Electrochemical (bio)sensors based on carbon cloth and carbon paper: An overview. Trends Anal Chem 142, 116324 (2021).

19. Khanna R, Cayumil R. Recent Developments in the Field of Carbon Fibers. BoD-Books on Demand (2018).

20. Saito N, et al. Application of carbon fibers to biomaterials: a new era of nano-level control of carbon fibers after 30-years of development. Chem Soc Rev 40, 3824-3834 (2011).

21. Forsythe RC, Cox CP, Wilsey MK, Müller AM. Pulsed Laser in Liquids Made Nanomaterials for Catalysis. Chem Rev 121, 7568-7637 (2021).

22. Faunce T, et al. Artificial photosynthesis as a frontier technology for energy sustainability. Energy Environ Sci 6, 1074-1076 (2013).

23. Ardo S, et al. Pathways to electrochemical solar-hydrogen technologies. Energy Environ Sci 11, 2768-2783 (2018).

24. Bhat ZM, et al. An electrochemical neutralization cell for spontaneous water desalination. Joule 4, 1730-1742 (2020).

25. Shi X, Back S, Gill TM, Siahrostami S, Zheng X. Electrochemical Synthesis of H2O2 by Two-Electron Water Oxidation Reaction. Chem 7, 38-63 (2021). 
26. Yuan C-J, Wang C-L, Wu TY, Hwang K-C, Chao W-C. Fabrication of a carbon fiber paper as the electrode and its application toward developing a sensitive unmediated amperometric biosensor. Biosens Bioelectron 26, 2858-2863 (2011).

27. Chen JYC, et al. Operando Analysis of NiFe and Fe Oxyhydroxide Electrocatalysts for Water Oxidation: Detection of Fe4+ by Mössbauer Spectroscopy. J Am Chem Soc 137, 15090-15093 (2015).

28. Kong D, Wang H, Lu Z, Cui Y. CoSe2 Nanoparticles Grown on Carbon Fiber Paper: An Efficient and Stable Electrocatalyst for Hydrogen Evolution Reaction. J Am Chem Soc 136, 4897-4900 (2014).

29. Cai X, et al. Binary metal sulfides and polypyrrole on vertically aligned carbon nanotube arrays/carbon fiber paper as high-performance electrodes. J Mater Chem A 3, 22043-22052 (2015).

30. Liu Z, Tang C, Chen P, Yu Q, Li W. Modification of carbon fiber by air plasma and its adhesion with BMI resin. RSC Adv 4, 26881-26887 (2014).

31. Fu X, Lu W, Chung D. Ozone treatment of carbon fiber for reinforcing cement. Carbon 36, 1337-1345 (1998).

32. Kim H, Lee Y-J, Lee D-C, Park G-G, Yoo Y. Fabrication of the carbon paper by wetlaying of ozone-treated carbon fibers with hydrophilic functional groups. Carbon 60, 429-436 (2013).

33. Zhang $\mathrm{H}$, et al. Surface engineering of carbon fiber paper for efficient capacitive energy storage. J Mater Chem A 4, 18639-18645 (2016).

34. Alvar EN, Zhou B, Eichhorn SH. Oxidative Treatment to Improve Coating and Electrochemical Stability of Carbon Fiber Paper with Niobium Doped Titanium Dioxide Sols for Potential Applications in Fuel Cells. Electrochim Acta 132, 347-355 (2014).

35. Deng F, et al. Synthesis of ultrathin mesoporous NiCo2O4 nanosheets on carbon fiber paper as integrated high-performance electrodes for supercapacitors. J Power Sources 251, 202-207 (2014).

36. Yang C, Shen J, Wang C, Fei H, Bao H, Wang G. All-solid-state asymmetric supercapacitor based on reduced graphene oxide/carbon nanotube and carbon fiber paper/polypyrrole electrodes. J Mater Chem A 2, 1458-1464 (2014).

37. Suktha P, et al. High-Performance Supercapacitor of Functionalized Carbon Fiber Paper with High Surface Ionic and Bulk Electronic Conductivity: Effect of Organic Functional Groups. Electrochim Acta 176, 504-513 (2015). 
38. Ham YS, Park YS, Jo A, Jang JH, Kim S-K, Kim JJ. Proton-exchange membrane CO2 electrolyzer for $\mathrm{CO}$ production using Ag catalyst directly electrodeposited onto gas diffusion layer. J Power Sources 437, 226898 (2019).

39. Wang Z, et al. Activated carbon fiber paper with exceptional capacitive performance as a robust electrode for supercapacitors. J Mater Chem A 4, 5828-5833 (2016).

40. Kazemi SH, Malae K. Electrodeposited $\mathrm{Ni}(\mathrm{OH}) 2$ nanostructures on electro-etched carbon fiber paper for highly stable supercapacitors. J Iran Chem Soc 14, 419-425 (2017).

41. Yi Y, et al. Electrochemical corrosion of a glassy carbon electrode. Catal Today 295, 3240 (2017).

42. Nigrovski B, et al. The influence of microwave heating on the texture and catalytic properties of oxidized multi-walled carbon nanotubes. Catal Commun 10, 1473-1477 (2009).

43. Datsyuk V, et al. Chemical oxidation of multiwalled carbon nanotubes. Carbon 46, 833840 (2008).

44. Utsumi S, et al. Direct evidence on $\mathrm{C}-\mathrm{C}$ single bonding in single-wall carbon nanohorn aggregates. J Phys Chem C 111, 5572-5575 (2007).

45. Desimoni E, Casella G, Morone A, Salvi A. XPS determination of oxygen-containing functional groups on carbon-fibre surfaces and the cleaning of these surfaces. Surf Interface Anal 15, 627-634 (1990).

46. Lesiak B, et al. $\mathrm{C} \mathrm{sp2/sp3} \mathrm{hybridizations} \mathrm{in} \mathrm{carbon} \mathrm{nanomaterials} \mathrm{-} \mathrm{XPS} \mathrm{and} \mathrm{(X)AES}$ study. Appl Surf Sci 452, 223-231 (2018).

47. Barclay M, Hill SB, Fairbrother DH. Use of X-ray photoelectron spectroscopy and spectroscopic ellipsometry to characterize carbonaceous films modified by electrons and hydrogen atoms. Appl Surf Sci 479, 557-568 (2019).

48. Kovtun A, Jones D, Dell'Elce S, Treossi E, Liscio A, Palermo V. Accurate chemical analysis of oxygenated graphene-based materials using X-ray photoelectron spectroscopy. Carbon 143, 268-275 (2019).

49. Estrade-Szwarckopf H. XPS photoemission in carbonaceous materials: A "defect" peak beside the graphitic asymmetric peak. Carbon 42, 1713-1721 (2004).

50. Johansson G, Hedman J, Berndtsson A, Klasson M, Nilsson R. Calibration of electron spectra. J Electron Spectrosc Relat Phenom 2, 295-317 (1973).

51. NIST X-ray Photoelectron Spectroscopy Database. X-ray Photoelectron Spectroscopy Database 20, Version 4.1 (National Institute of Standards and Technology, Gaithersburg, 2012); http://srdata.nist.gov/xps/. 
52. Barr TL, Seal S. Nature of the use of adventitious carbon as a binding energy standard. $J$ Vac Sci Technol A 13, 1239-1246 (1995).

53. Yumitori S. Correlation of $\mathrm{C} 1 \mathrm{~s}$ chemical state intensities with the O1s intensity in the XPS analysis of anodically oxidized glass-like carbon samples. J Mater Sci 35, 139-146 (2000).

54. Smith M, Scudiero L, Espinal J, McEwen J-S, Garcia-Perez M. Improving the deconvolution and interpretation of XPS spectra from chars by ab initio calculations. Carbon 110, 155-171 (2016).

55. Morgan DJ. Comments on the XPS Analysis of Carbon Materials. C 7, 51 (2021).

56. Desimoni E, Casella GI, Salvi AM, Cataldi TRI, Morone A. XPS investigation of ultrahigh-vacuum storage effects on carbon fibre surfaces. Carbon 30, 527-531 (1992).

57. Trotochaud L, Young SL, Ranney JK, Boettcher SW. Nickel-Iron Oxyhydroxide Oxygen-Evolution Electrocatalysts: the Role of Intentional and Incidental Iron Incorporation. J Am Chem Soc 136, 6744-6753 (2014).

58. Shirley DA. High-Resolution X-Ray Photoemission Spectrum of the Valence Bands of Gold. Phys Rev B 5, 4709-4714 (1972). 


\section{Supplementary Files}

This is a list of supplementary files associated with this preprint. Click to download.

- 220121SIHydrophilicCFPAMM.pdf 